\title{
Chapter 7 \\ The DIALLS Platform: Supporting Cultural Literacy and Understanding of European Values Over the Internet
}

\author{
Lucas M. Bietti, Ben Zion Slakmon, Michael J. Baker, Françoise Détienne, \\ Stéphane Safin, and Baruch B. Schwarz
}

\subsection{Introduction}

In this chapter we present the process of designing and developing a novel online platform for supporting cultural literacy learning, involving the elaboration and understanding of European values in collaborative dialogue between students, with teacherled reflection on wordless texts. Wordless texts are books or videos that comprise sequences of pictures which stimulate student readers to reconstruct the attendant narratives (see Chapters 5 and 6, this volume). The narratives in question, available publicly, are designed to stimulate discussions relating to European values, notably tolerance, empathy and inclusion (Lähdesmäki et al. 2020). The main questions for platform design were therefore how to facilitate productive discussions involving European values, on or around such wordless texts, and to structure such discussions in a way that is closely anchored in the texts.

Computer Supported Collaborative Learning (CSCL) systems can support and guide productive dialogue and argumentation (Andriessen et al. 2003; Schwarz and Baker 2017), leading to deeper understanding in the sense of knowledge building (Bereiter and Scardamalia 2003), or in the sense of knowing the other across cultural

L. M. Bietti ( $\varangle)$

Norwegian University of Science and Technology, Trondheim, Norway

e-mail: lucas.bietti@ntnu.no

L. M. Bietti · M. J. Baker · F. Détienne

Centre National de La Recherche Scientifique, Paris, France

B. Z. Slakmon

Tel Aviv University, Tel Aviv, Israel

M. J. Baker · F. Détienne · S. Safin

Télécom Paris, Paris, France

B. B. Schwarz

Hebrew University of Jerusalem, Jerusalem, Israel

(C) The Author(s) 2021

F. Maine and M. Vrikki (eds.), Dialogue for Intercultural Understanding, https://doi.org/10.1007/978-3-030-71778-0_7 
differences (Wegerif et al. 2017). CSCL systems can play an important role in such learning to the extent that they enable task sequences and interpersonal communication media to be structured in ways that favour the co-elaboration of knowledge (e.g. Dillenbourg 1999).

The DIALLS Platform facilitates dialogue both within countries and across them, as well as the sharing, use and co-creation of cultural resources (such as multimodal texts, images, videos), thereby opening up various pedagogical opportunities for building a shared culture. The objective of the DIALLS Platform is to support the educational activities proposed by the project (see overview in Chapter 1), and to meet user needs. Three categories of users were defined: (1) students (at three age groups; 5-6, 8-9 and 14-15 years old), across the project participants' countries; (2) teachers, relating to 1 ; and (3) researchers, analyzing data from use of the DIALLS platform.

The design of the platform thus faced a number of specific challenges. The first related to the need to provide a platform that was usable across a broad age-range of students, from 5 to 15 years old. Depending on the age, language abilities and the roles of teachers in mediating discussions are quite varied. The second challenge was the need to focus and structure discussions on and around sequences of images, still or animated, comprising the wordless text. Thirdly, use of the platform across several EU countries (e.g. UK, Lithuanian, Portugal, Cyprus, Portugal, Spain, Germany), as well as Israel, of course raises the thorny 'language problem'. Given the broad age range, most students can not be expected to discuss in a language that is not their own native language; and yet, reliable automatic translation does not exist for all of the project languages, especially with respect to free input, on chat systems, that would bear many traces of everyday and SMS language.

Our general approach (Fig. 7.1) to the design and development of the DIALLS Platform was user centered (Sharp et al. 2019). Indeed, users of the platform -i.e. teachers, researchers and learners-, were involved in the design phase as well as in the evaluation phase. Following an analysis of the DIALLS educational concept as

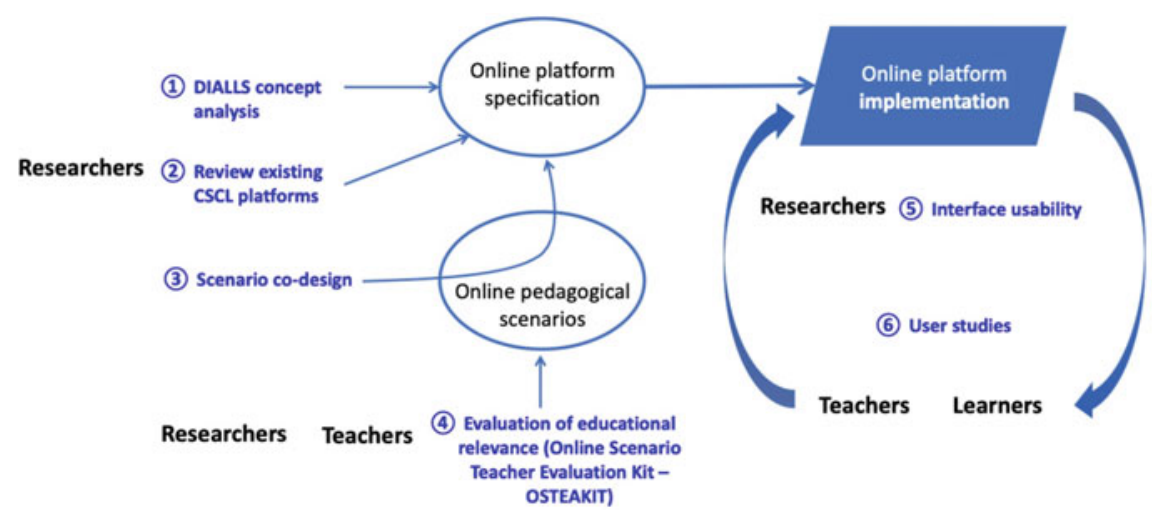

Fig. 7.1 General approach taken for the design and development of the DIALLS platform 
well as a detailed literature review of relevant CSCL research, which provided a preliminary identification of the functions of the platform, a scenario-based design phase (Carroll 1997) was conducted in co-design workshops involving teachers and researchers. The objective was to further refine the functionalities of the platform in order to fit users' requirements. In the evaluation phase, two kinds of methods were mobilized: (1) user studies involving teachers and researchers aimed at evaluating the educational relevance and usability of the platform; (2) an expert inspection method (Scapin and Bastien 1997) aimed at evaluating the interface usability (this step is not detailed in this chapter). Finally, user studies with teachers and learners have been carried out in order to further adapt the platform to use in classrooms.

In this chapter, we first present a systematic review of existing computer tools that support the co-creation of multimedia teaching resources on the basis of the functionalities described in the DIALLS user requirements document (Sect. 7.2). The aim of such a systematic review was to identify the specific functions of existing CSCL systems that could be relevant for the development and design of the DIALLS Platform. The co-design workshops with expert users (researchers and teachers) of the DIALLS Platform are reported in Sect. 7.3. The aim of the co-design workshops was to further specify the functionalities of the platform, by envisioning the tools that would be required to support computer mediated DIALLS activities (i.e. online pedagogical scenarios). In Sect. 7.4, we introduce a description of the main functionalities of DIALLS platform and its interface that corresponds to the version of the platform tested by teachers in June and July 2019.

In Sect. 7.5, we present the user studies with teachers who were recruited to implement the DIALLS educational program in their classroom. User studies were conducted in Cyprus, Germany, Lithuania, Spain, and the United Kingdom and provided essential information for the incorporation of successive changes to the platform before its implementation in Europe and Israel in the second part of the DIALLS project. In Sect. 7.6 we present some general reflections on the entire design process and include some recommendations for the development of similar computer tools in large interdisciplinary research projects. In the last section (Sect. 7.7) we discuss the importance of applying a comprehensive socio-technical system approach to the design and development of new educational technology for innovative pedagogical and societal objectives.

\subsection{Systematic Review}

The aim of our systematic review of the literature and the CSCL systems supporting dialogue and argumentation was to provide initial insight into the computer tools (e.g. synchronous CHAT tools) that the DIALLS Platform should incorporate in order to support the educational activities that are part of the DIALLS project and its educational concept (see Chapter 1 in this book). Given that we aimed to reuse existing tools as far as possible in implementing the DIALLS platform, we also searched for CSCL tools that were still available for download and installation, including, if possible, 
publicly available source code. In May 2018 we searched for systematic reviews of CSCL systems supporting dialogue and argumentation published between 2010 and 2018. Two digital libraries were searched: the library of the University of ParisSaclay and Scopus. Searches were facilitated using four sets of keywords covering "CSCL", "argumentation", "software", "program(me)", and "dialogue". We ended with 72 research articles that were published in academic journals and peer-reviewed conference proceedings. Research articles included empirical studies that evaluated 34 CSCL systems supporting dialogue and collaborative argumentation. We decided to exclude eight CSCL systems from our review because they were only tested with university students, whereas our own student users are in primary and secondary schools. We employed inductive and deductive methods for the analysis of the literature. Inductive methods were used for the identification of the basic characteristics shared by most the CSCL systems included in the corpus. Deductive methods were employed for the analysis of the CSCL tools that would support the required functionalities of the DIALLS Platform. We paid particular attention to the following five computer functionalities whose importance for the DIALLS Platform we considered to be essential, described below: (i) Session set-up; (ii) Text display; (iii) Online discussion; (iv) Document repository; and (v) Annotation. We applied these five thematic categories to each of 72 research articles reporting the use and assessment of CSCL systems included in the corpus.

Session set-up. Teachers have to initiate sessions and invite learners to join those sessions. Teachers and learners have to choose nicknames and passwords as an authentication mode and to create their user identities. Log-on management, session creation and document upload tools are the most frequent features reported in the literature. Limited studies have examined how users interact with log-on management functionalities in computer systems (Kim et al. 2007). Kim et al. have observed that primary school students experienced problems when using the log-on management function. Some children could not recall their identification numbers and passwords, which resulted in increasing feelings of frustration and blockage. For this reason (amongst others), primary school students are not required to log onto the DIALLS platform, their discussions being mediated by their teachers.

Text display. These are tools that learners use to have access to texts, video and multiple forms of media included in the activities. They also enable teachers to send talk prompts to learners whenever needed. Teachers' use of prompts (e.g., wordless texts) have positively affected students' discussions and engagement with the activities (Kim et al. 2007). These are CSCL functionalities that are generally taken for granted in the literature; therefore, there is limited information on teachers' and students' user experience in the corpus we selected for the review. However, CSCL systems such as ARGUNAUT (Schwarz and Asterhan 2011) and CoFFEE (Belgiorno et al. 2008) contain all or some of those functionalities (the video display tool can be found in more recent software only).

Online discussion. Online forums for asynchronous discussions and synchronous chat are the most common online dialogue and discussion tools found in CSCL systems. CSCL systems supporting asynchronous communication generally support an online forum only whereas those supporting synchronous and asynchronous 
communication (e.g. CoFFEE) support an online forum and a CHAT system (see Talkwall, Ludvigsen et al. 2020). Kim and colleagues found that primary school students experienced problems using online forums due to low proficiency in keyboarding or use of the computer systems, and some were concerned about spelling errors (Kim et al. 2007). This finding has been validated by studies that used other computer systems too (Prinsen et al. 2007). Low proficiency in keywording and writing skills required frequent interventions from research assistants that interfered with the progression of the task. With respect to secondary school students, research has focused on the benefits of using synchronous CHAT compared to face-to-face dialogue in collaborative argumentation situations (Baker et al. 2007).

Document repository. These tools allow users to store and share text and image files. Document repositories are tools largely supported by most of the $26 \mathrm{CSCL}$ systems included in the review. However, its systematic assessment in the literature seems to be rather limited. With respect to primary school students, document repositories have been used to store teachers' comments and feedback on quality of arguments produced by children in online discussion forums. CSCL systems such as Digalo, and CoFFEE allow teachers to upload wordless texts and instructions onto the platform in order to stimulate and structure students' discussions. For example, the document upload tool has been used to present questions to students for later reflection and discussion on the online forum between primary (Kim et al. 2007), and secondary (Ding 2009) students. Prinsen et al. (see below) have shown that a record of teachers' feedback on children's arguments facilitated students' appropriation of positive behaviours and inhibited the use of ineffective behaviours in future collaborative argumentation tasks (Prinsen et al. 2009). Another functionality investigated in the literature was the possibility for gathering data from the Internet for storage and later use in the online forum, which has been shown to positively affect group discussions (Clark et al. 2009).

Annotation. These are basic features in CSCL systems that are in line with the key premises in CSCL research presented above-i.e. annotation tools provide external scaffolds for learners to produce high quality discussion. Together with online discussion tools (see above), annotation tools have been the most evaluated features of CSCL systems. Forty-eight empirical studies included in our corpus dealt with annotation tools. Research has shown that highlighting relevant portions of dialogues and arguments may help students easily identify parts of the solution they need to pay special attention to (Dragon et al. 2006).

After carefully reviewing the literature and the computer tools that each of the 26 CSCL systems included in the review encompass, it was found that there is no single existing system that satisfies all the requirements of the DIALLS Platform. CoFFEE and Talkwall were the currently available systems that came closest to satisfying design requirements of the DIALLS Platform. However, these computer systems meet these requirements almost exclusively for older children, with little or consideration of teacher-mediated discussions at primary school level. None of the CSCL systems included in the review contained a semi-automatic text translation option. This is a crucial feature for the DIALLS Platform to include considering its 
multi-cultural and multi-lingual perspective on computer-mediated cultural literacy learning in schools in Europe and Israel.

\subsection{Co-Design Workshops}

In September and November 2018, two co-design workshops with future users of the DIALLS Platform (researchers and teachers) were held in Paris and Cambridge, respectively. Co-design workshops were of crucial importance at the beginning of the design of the DIALLS platform, for anticipating future educational activities to be supported. Workshop participants were asked to collaboratively design educational activities for the classroom in order to provide a detailed contextualization of the CSCL system functionalities analyzed in the review and how they could be adapted to the DIALLS project.

The co-design workshop technique proposed for the development of the DIALLS platform was rooted in the concept of scenario-based design (Carroll 1997). The aim is to contextualize design proposals in a narrative form at the beginning of projects. This is important because, as the design of the DIALLS platform progressed, the possibilities for changes and modification decreased. Therefore, the co-design workshop technique provided a space where potential users of the DIALLS Platform could collaborate in the co-construction of design features of the platform and test out different ideas in a simulated environment. This occurred before designers and developers made irreversible technical choices.

The co-design workshops were developed to ensure that future users of the DIALLS Platform would be able to co-construct detailed educational scenarios that would resemble those included in the actual foreseen classroom activities. Co-design workshops were organized following the DIALLS core educational activities. These involve (1) observing and interpreting wordless texts (framed by the teacher) and (2) discussing interpretations in order to co-create meanings/interpretations.

The user-centered activity scenarios produced by DIALLS researchers during the co-design workshop had some general features depending on each of the specific age groups included in the DIALLS project. Researchers and teachers agreed on the fact that the DIALLS Platform should come with a user-friendly interface. Users would prefer to interact with a relatively easy-to-learn-and-use interface that would not require them to participate in an excessively long training program. They indicated that for pre-primary (5-6 years old) and primary school (8-9 years old) children, the DIALLS Platform was used as a tool to connect classrooms in different countries and display cultural artefacts produced by students in order to promote collective reflection and dialogue. However, in DIALLS teachers play a key role in the collaborative activities between classrooms, whilst most existing CSCL systems (e.g. synchronous CHAT) consider students to be the primary main users. This led to a further specification of the functionalities of the DIALLS Platform for supporting 5-6 and 89 years old. In the user-centered activity scenarios for older students, teachers play a limited role in the individual and collaborative activities, in the classroom and 
in between classrooms. Finally, workshop participants indicated that the DIALLS Platform should contain tools to support the semi-automatic translation of text when classrooms from different countries were participating in online discussions.

Co-design workshops provided a detailed contextualization of the CSCL system functionalities analyzed in the critical review and how they could be adapted and refined for the DIALLS Platform. Co-design workshops led to our functional recommendations of the list computer tools that the DIALLS Platform should contain. Both co-design workshops have served to validate the tools that we retained from existing CSCL systems and to propose new ones in order to support the specificities of the DIALLS project. The user-centered activity scenarios created by DIALLS researchers and teachers allowed us to further specific those tools and to adapt them to the particular age groups.

\subsection{The DIALLS Platform}

In this section we present the main functionalities of the DIALLS platform that teachers across the project consortium tested in June and July 2019 (Fig. 7.2). The general description of the platform is organized into the following categories: users; language support; resources; features; training; and data privacy and protection.

Users. The platform supports two types of users: teacher and student.

Language support. The system has been designed to support any language, including Semitic languages (right to left writing systems).

Resources. The platform supports three types of resources: images (PDF, JPG, PNG and TIFF files); texts (PDF and Word files); and films (MP4 and MOV files).

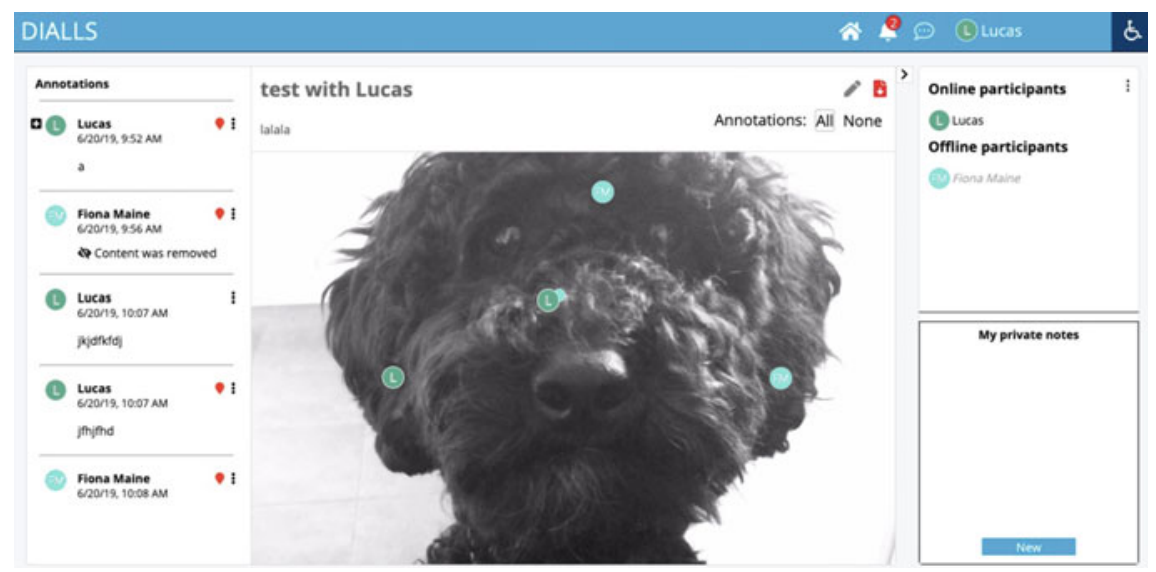

Fig. 7.2 DIALLS Platform interface: image discussed (middle); annotations/participants (left/right) 
Features. The platform includes several features: discussion rooms; annotation tools; resource management tools; and separate box for notes. In discussion rooms, a teacher can create a new discussion based on resources and select the participants from the DIALLS list of users. A teacher may choose the discussion's age group, and thereby adjust the interface and font size accordingly. Users (teachers and students) can start a discussion on any part of the uploaded resources and comment on any discussion in which they are participating. Any discussion has a start time and may have an end date. Once the end date has passed the discussion state is set to be "closed" (a "closed discussion" becomes read-only). Then, the discussion that is relevant for a specific user can be seen on the users' dashboard. Annotations are posts in a threaded discussion. A user may choose to start a new discussion and annotate on a specific area of the discussion resource. She can pick the relevant point and start a new discussion from there or write the annotation and then link it to the relevant point on the uploaded resources. In addition, a user may decide to post a comment or start a discussion without being anchored in the resource being discussed in the discussion room - this is particularly important for the phase of discussion where the students move from reconstructing the narrative to a more general discussion on the ethical questions and concepts at stake. A teacher can use resource management tools to upload and manage resources on the platform. The actions that teachers can perform include: uploading a new resource; sharing a resource with the DIALLS platform for broader use; saving a link as resource; and utilizing the platform library of shared resources to create new discussion rooms. Notes are initially personal and are not seen by the other participants of the discussion. Any user can write her own notes, and these can be found on a "notes" widget in the specific page in which it was created. Notes can be related to a specific area in the uploaded resource for discussion and/or particular comments. This is not a requirement as notes can also be without any discussion context. Finally, notes may be published and become part of the discussion and thereby seen by the other participants in the discussion room.

Training. Online training workshops were offered to DIALLS researchers in 2019 and 2020 in the form of video tutorials addressing specific needs (e.g. adjusting contents to digital literacy skills of teachers and students across the consortium).

Data privacy and protection. The platform meets European GDPR (The General Data Protection Regulation) requirements, which include the anonymization of private users, users' data retrieval and users' data removal. The system contains an automatic anti-virus tool for newly uploaded resources.

\subsection{User Studies with Teachers}

\subsubsection{Online Scenario Teacher Evaluation}

In this section we present the user-study (hereafter, "Online Scenario Teacher Evaluation Kit"-OSTEAKIT) that we designed and coordinated to test (i) the functions 
of the DIALLS platform as described in Sect. 7.4 and (ii) a subset of blended online pedagogical scenarios with teachers involved in the DIALLS project. These blended online pedagogical scenarios were based on lesson plans as developed by project partners. The transformation of lesson plans into online scenarios followed the general guidelines set in the co-design workshops held in Paris and Cambridge.

Our goal with OSTEAKIT was to obtain as much feedback as possible on the DIALLS platform and the proposed blended online pedagogical scenarios. Pedagogical scenarios are teaching and learning models that present scripts of what students and teachers should do when they participate in educational activities (Andriessen and Sandberg 1999). Such scripts include a set of activities, methods and resources that are used to introduce students to concepts and processes in relation to specific learning objectives (Wichmann et al. 2010). Pedagogical scenarios provide meaningful and ecologically valid contexts for the achievement of those objectives. They are used to create learning situations with well-defined roles, activities, sequences of actions, resources and tools (Wichmann et al. 2010). Thus, pedagogical scenarios scaffold educational activities and enable students to accomplish learning objectives. Pedagogical scenarios can encompass different levels of detail which are determined by specific educational goals.

User studies were coordinated locally, by DIALLS researchers in Cyprus, Germany, Lithuania, Portugal and the United Kingdom between June and July 2019. OSTEAKIT included six blended online pedagogical scenarios, three for synchronous lessons (two classes in the same country/language) and another three for asynchronous lessons (two classes in different countries/languages). We added two online scenarios for each age group, that is one synchronous and asynchronous blended online pedagogical scenarios for students aged 5-6, 8-9 and 14-15 years old respectively. They combined and integrated face-to-face teaching and learning approaches with activities that may involve, to a greater and lesser extent the use of online information and communication technologies.

OSTEAKIT also contained a general System Usability Scale (SUS) questionnaire that we used to measure the usability of the DIALLS platform in relation to the teachers' general user experience with the platform (e.g. interface design, consistency and simplicity) and a questionnaire related to the blended online pedagogical online scenarios. SUS is a standardized 10-item Likert scale questionnaire that provides an at-a-glance look at the ease of use (or lack thereof) of the DIALLS platform. At the end of the questionnaire there was a section where teachers could add any comments they had on the DIALLS platform. The aim of the questionnaire specifically related to the blended online pedagogical scenarios was to obtain as much feedback as possible about their educational value within the framework of the DIALLS project. In addition, the questionnaire invited teachers to suggest changes to the online scenarios and propose alternative ways of using the DIALLS platform in the form of new scenarios or new functionalities to support.

Teachers $(n=21)$ were familiar with the general DIALLS concept as they were part of the pool of teachers who were recruited to use the platform in their classrooms. Thus, we ensured that teachers participating in the DIALLS project and project partners were actively involved in the design and development of the online tools 
supported by the DIALLS platform and the blended online pedagogical scenarios that the same teachers would use with the students. Such active involvement also enabled researchers and teachers to be introduced to the platform's functionalities.

Teachers' responses explained what functions should be added and how existing tools could be modified to improve the platform so it could support the pedagogical goals of the project.

They mentioned that they would want the platform to have new tools such as a separate chat tool, a tool that would allow them to upload multiple resources, not only PDF files. Some teachers' responses manifested a general concern about the necessity of receiving more specific training about how to use it properly to achieve the pedagogical goals contained in synchronous and asynchronous lessons. However, others reported not needing additional training in general IT skills and that they believed they could use the DIALLS platform without the support of technical personnel. Teachers' responses at the country level showed some of the diversity in teachers' experiences with information and communication technologies, and probably the impact of different cultural and institutional practices with regards to the use of technology in the classroom. Differences in teachers' responses at the country level were used for the design of highly targeted training materials, including more specific teachers' guides for the use of the platform, online training sessions and video tutorials.

Additionally, teachers referred to the different ways to improve the blended online pedagogical scenarios. Teachers expressed issues with time management in the sense that using the platform and completing the blended online scenarios was too time consuming. Hence, they suggested limiting the use of the platform to particular tasks (e.g. sharing and commenting on cultural artifacts) and that teachers/students should try to avoid creating several discussion rooms. Teachers also suggested that more time could be allocated to face-to-face class discussion and that the blended online pedagogical scenarios should promote increased physical manipulation of picture books and allocate more time for the students' selection of features in them that they consider to be relevant and worth exploring.

\subsubsection{Addressing Cultural and Linguistic Diversity}

One of the DIALLS project's strengths and innovative approaches is that it takes into consideration the cultural and linguistic diversity of Europe and beyond. This also creates important challenges, notably, the "language problem": how can teachers and students engage in cross-national discussions over Internet, who have different native languages (in the case of DIALLS: English, Lithuanian, Hebrew, Greek, German, Portuguese, Spanish and Catalan)? Teachers expressed that online automatic translation software only would not be a suitable option for translations in asynchronous and expressed the possibility of having teachers writing messages in English only in those lessons. In order to solve this issue, we developed four translation strategies that DIALLS partners may want to consider for translating dialogue traces as result 
of interactions between classes located in different countries, to be used partially or conjointly in a flexible way, depending on the situation (see Fig. 7.3).

1. Technical: This strategy involves the use of existing tools, such as Google Translate or similar tools available and free on the web include Microsoft Translator; Bing Translator; Yandex.Translate; Babylon; Apertium; and DeepL Translator. This strategy is highly dependent on the language and pairs of languages concerned.

2. Socio-technical: This strategy lies in the use of the linguistic expertise in the immediate environment of the partners, to do human translation and/or checking of automatic translation (see Strategy \#1).

3. Multi-lingual: This strategy involves the use of an "interlanguage" between classes located in different countries. That is, depending on specific pairs of partner countries whose school children are discussing, it may be possible to consider discussions in: (i) L2/L2 - e.g. schools in Portugal and Lithuania discussing in English, with foreign language classes/teachers; (ii) L1/L2 e.g. schools in England and in Cyprus discussing in English. The priority should be communication and mutual understanding rather than grammatical correctness.
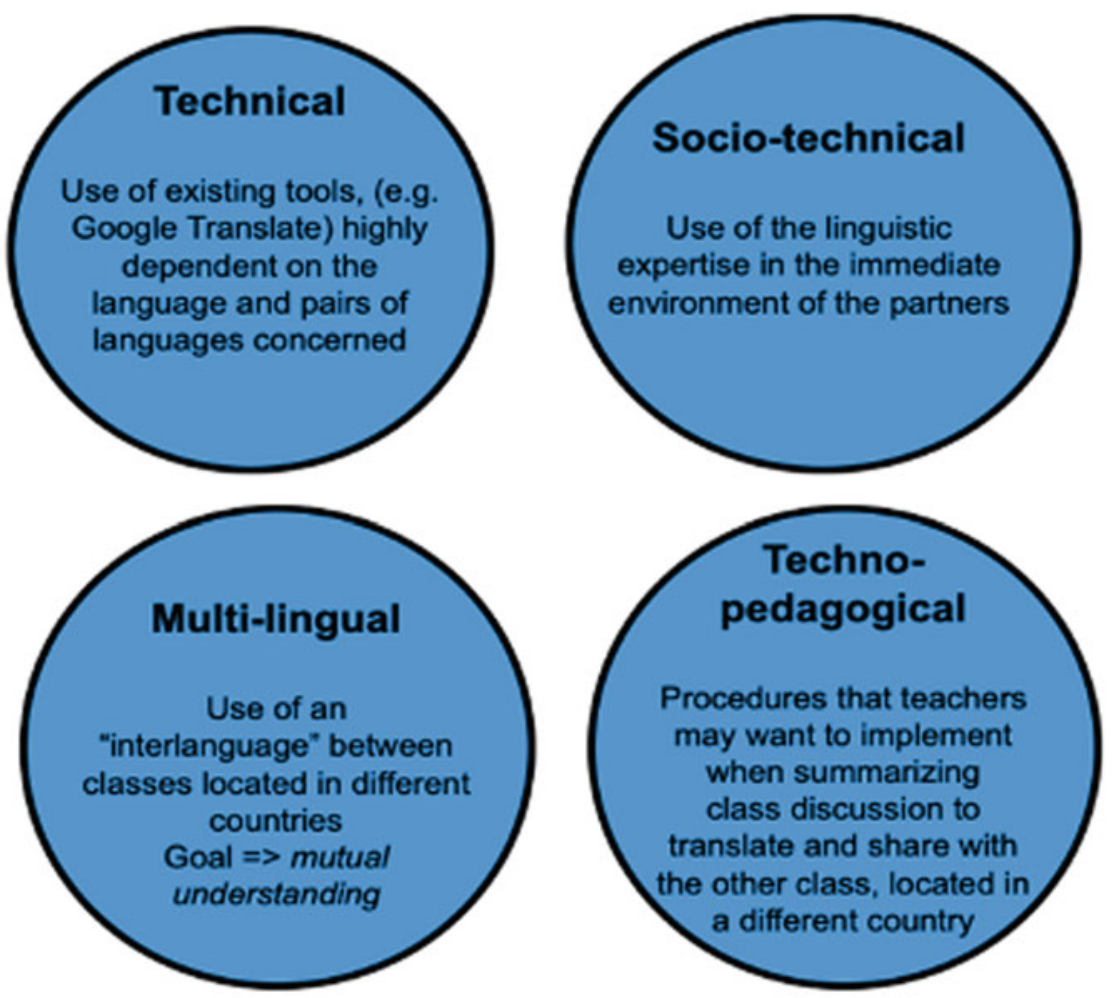

Fig. 7.3 Translation strategies for dialogue tracing 
4. Techno-pedagogical: This strategy involves trying to reconcile technological limitations - for example, absence of automatic translation tools for a pair of languages - and pedagogical affordances. Thus, asking students in each country, or even their teachers, in the case of younger children, to write a short synthesis of their chat discussions can at the same time facilitate translation (e.g. via the socio-technical approach) and also be a pedagogically valuable activity, facilitating knowledge integration.

\subsection{Reflections}

The design of the DIALLS platform came with important challenges. One of the most important of them was the management of the expectations of members of a large interdisciplinary research group. The research partners responsible for the design and development of the computer tool (Hebrew University of Jerusalem and CNRS Paris) learned that it was essential to discuss with project partners who were not familiar with the field education technology what type of Internet-based platform could be developed considering multiple constraints. These constraints included technological constraints, budgetary constraints, and time constraints, amongst several others (e.g. GDPR data management and ethics regulations). Technological constraints determined what type of computer system we were able to realistically produce within the framework of the project. For example, the inclusion of an automatic translation function for the chat tool to support intercultural communication was far beyond the kind of technology and computational engineering knowledge we had at our disposal. Researchers who are not familiar with the field of education technology may assume that technological advancements such as the Google Translate tool might be developed for computer tools in education, or otherwise be easily integrated as embedded applications into system. Technological constraints are very much related to budgetary constraints. The design of most of the freely available Internet tools (e.g. video-chat tool) we use, without much effort, on a regular basis were developed by some of the largest corporations in the world. Such commonly used Internet tools shape researchers' expectations regarding what research partners working in CSCL in a scientific project could produce. Time constraints were also crucial; and despite being intrinsically related to technological and budgetary constraints, they are also given by the time schedule of the planned activities in large research projects. For example, in the DIALLS project the time spent on the development of the computer tool was determined by institutional agreements with hundreds of primary and secondary schools indicating the period when data could be collected in the classrooms. In order to better manage users expectations it is crucial to have open discussions about issues like these at the beginning of the endeavour. 


\subsection{Conclusion}

In this chapter we presented a detailed description of the general approach we undertook to design a new Internet-based platform (DIALLS platform) for supporting cultural literacy and understanding of European values based on collaborative and teacher-led reflection on wordless texts (Fig. 7.1). The design and development of a novel online platform included a (i) systematic review of existing computer systems in CSCL, co-design workshop with researchers and teachers involved in the DIALLS project, (ii) the design and development of the platform and (iii) exhaustive user studies with teachers who were familiar with the DIALLS goals. Most research in CSCL focuses on the evaluation of usage of systems, giving few details of the initial, possibly collaborative system design process. We have shared general reflections on the processes of design and development of the DIALLS platform, designed for a specific and original educational purpose, within a large interdisciplinary research project. We included some recommendations based on our experience, namely being realistic about the types of functions the computer tool may afford and having open discussions about them from the beginning of the project.

Our challenge was to make sure that tools and structures provided by the DIALLS Platform would on one hand support and yet not limit the pedagogical diversity involved in the DIALLS project. The tools of the DIALLS Platform enabled its users to analyze the dynamic development of cultural values (e.g., tolerance and inclusion) in dialogue. Thus, the structures provided by the platform support such fluid dialogue that allows building on other students' responses, elaborating and justifying ideas and synthetizing group thinking processes.

We proposed that the most adequate way to address users' expectations with the DIALLS platform was to adopt a socio-technical system approach (Cooper and Foster 1971), involving a web of mutual dependencies between users, other technologies, and cultural and institutional practices. This has enabled meeting two challenges that, to our knowledge, have not been addressed by existing CSCL research, based on concurrent co-design of educational scenarios and tools: designing and developing a system that is adapted to achieving specific educational goals (cultural literacy learning) across very different age groups of students, and overcoming the language barrier in cross-country collaboration.

\section{References}

Andriessen, J., and J. Sandberg. 1999. Where is education heading and how about AI? International Journal of Artificial Intelligence in Education 10 (2): 130-150.

Andriessen, J., M.J. Baker, and D. Suthers, eds. 2003. Arguing to learn: Confronting cognitions in computer-supported collaborative learning environments. Dordrecht: Kluwer.

Baker, M.J., J. Andriessen, K. Lund, M. van Amelsvoort, and M. Quignard. 2007. Rainbow: A framework for analysing computer-mediated pedagogical debates. International Journal of Computer Supported Collaborative Learning 2 (2-3): 315-357. 
Beaver, J.K., B. Hallar, and L. Westmass. 2014. Blended learning: Defining models and examining conditions to support implementation. PERC Research Brief. Philadelphia: Research for Action.

Belgiorno, F., R. De Chiara, I. Manno, and V. Scarano. 2008. A flexible and tailorable architecture for scripts in F2F collaboration. In Times of convergence. Technologies across learning contexts. EC-TEL 2008. Lecture Notes in Computer Science, vol. 5192, ed. P. Dillenbourg and M. Specht, 401-412. Berlin and Heidelberg: Springer.

Bereiter, C., and M. Scardamalia. 2003. Learning to work creatively with knowledge. In Unravelling basic components and dimensions of powerful learning environments, ed. E. Corte, L . Verschaffel, N. Entwistle, and J. van Merriënboer, 55-68. EARLI Advances in Learning and Instruction Series. Bingley, UK: Emerald.

Carroll, J.M. 1997. Scenario-based design. In Handbook of human-computer interaction, ed. M.G. Helander, T.K. Landauer, and P.V. Prabhu, 383-406. Amsterdam: North Holland/Elsevier.

Clark, D.B., C.M. D'Angelo, and M. Menekse. 2009. Initial structuring of online discussions to improve learning and argumentation: Incorporating students' own explanations as seed comments versus an augmented-preset approach to seeding discussions. Journal of Science Education and Technology 18 (4): 321-333.

Cooper, R., and M. Foster. 1971. Sociotechnical systems. American Psychologist 26: 467-474.

Dillenbourg, P. 1999. Collaborative learning: Cognitive and computational approaches. Amsterdam: Pergamon/Elsevier Science.

Ding, N. 2009. Visualizing the sequential process of knowledge elaboration in computer-supported collaborative problem solving. Computers and Education 52 (2): 509-519.

Dragon, T., B.P. Woolf, D. Marshall, and T. Murray. 2006. Coaching within a domain independent inquiry environment. In Intelligent tutoring systems. ITS 2006. Lecture Notes in Computer Science, vol. 4053, ed. M. Ikeda, K.D. Ashley, and T.-W. Chan, 144-153. Berlin and Heidelberg: Springer.

Kim, I.-H., R.C. Anderson, K. Nguyen-Jahiel, and A. Archodidou. 2007. Discourse patterns during children's collaborative online discussions. Journal of the Learning Sciences 16 (3): 333-370.

Lähdesmäki, T., A.-K. Koistinen, and S.C. Ylönen. 2020. Intercultural dialogue in European education policies: A conceptual approach. New York: Palgrave Macmillan.

Ludvigsen, S.R., P. Warwick, I. Rasmussen, K.A. Rødnes, O. Smørdal, and L. Major. 2020. Learning as gap-closing. Investigating digitalized dialogues. In Designs for experimentation and Inquiry: Approaching learning and knowing in digital transformation, ed. Å. Mäkitalo, T.E. Nicewonger, and M. Elam. London: Routledge.

Polson, P. G., C. Lewis, J. Rieman, and C. Wharton. 1992. Cognitive walkthroughs: A method for theory-based evaluation of user interfaces. International Journal of Man-Machine Studies 36 (5): $741-773$.

Prinsen, F.R., M. Volman, and J. Terwel. 2007. The influence of learner characteristics on degree and type of participation in a CSCL environment. British Journal of Educational Technology 38 (6): 1037-1055.

Prinsen, F.R., M. Volman, J. Terwel, and P. Van den Eeden. 2009. Effects on participation of an experimental CSCL-programme to support elaboration: Do all students benefit? Computers and Education 52 (1): 113-125.

Scapin, D.L., and J.M. Bastien. 1997. Ergonomic criteria for evaluating the ergonomic quality of Interactive systems. Behaviour \& Information Technology 16 (4-5): 220-231.

Schwarz, B.B., and C.S. Asterhan. 2011. E-moderation of synchronous discussions in educational settings: A nascent practice. The Journal of the Learning Sciences 20 (3): 395-442.

Schwarz, B.B., and M.J. Baker. 2017. Dialogue, argumentation, and education. New York: Cambridge University Press.

Schwarz, B.B., and R. De Groot. 2007. Argumentation in a changing world. International Journal of Computer-Supported Collaborative Learning 2 (2-3): 297-313.

Sharp, H., J. Preece, and Y. Rogers. 2019. Interaction design: Beyond human-computer Interaction, 5 th ed. New York: John Wiley \& Sons. 
Suthers, D.D. 2003. Representational guidance for collaborative inquiry. In Arguing to learn: Confronting cognitions in computer-supported collaborative learning environments, ed. J. Andriessen, M.J. Baker, and D.D. Suthers, 27-46. Dordrecht: Kluwer.

Wegerif, R., J. Doney, A. Richards, N. Mansour, S. Larkin, and I. Jamison. 2017. Exploring the ontological dimension of dialogic education through an evaluation of the impact of internet mediated dialogue across cultural difference. Learning, Culture and Social Interaction 20: 80-89.

Wichmann, A., J. Engler, and U. Hoppe. 2010. Sharing educational scenario designs in practitioner communities. In Learning in the disciplines: Proceedings of the 9th international conference of the learning sciences (ICLS 2010), ed. K. Gomez, L.B. Lyons, and J. Radinsky, 750-757. Chicago IL: International Society of the Learning Sciences.

Lucas M. Bietti is an Associate Professor in psychology at the Norwegian University of Science and Technology. He holds an honorary affiliation with Centre National de la Recherche Scientifique at Télécom Paris.

Ben Zion Slakmon is an Assistant Professor of Education at Tel Aviv University.

Michael J. Baker is a tenured Research Professor in language sciences of the Centre National de la Recherche Scientifique at Télécom Paris. His research aims to understand the processes of collaborative learning and work, drawing on dialogue and argumentation analysis.

Françoise Détienne is a tenured Research Professor in cognitive ergonomics of the Centre National de la Recherche Scientifique at Télécom Paris. Her research focuses on technologymediated collaboration, co-design and online epistemic communities.

Stéphane Safin is an Associate Professor in cognitive ergonomics, at Télécom Paris. His research is on the role of technology mediation in group design and creativity.

Baruch B. Schwarz is a Professor of Education at the Hebrew University of Jerusalem. His research focuses on learning in social interaction, especially within the context of collaborative learning and argumentation in various domains of knowledge.

Open Access This chapter is licensed under the terms of the Creative Commons Attribution 4.0 International License (http://creativecommons.org/licenses/by/4.0/), which permits use, sharing, adaptation, distribution and reproduction in any medium or format, as long as you give appropriate credit to the original author(s) and the source, provide a link to the Creative Commons license and indicate if changes were made.

The images or other third party material in this chapter are included in the chapter's Creative Commons license, unless indicated otherwise in a credit line to the material. If material is not included in the chapter's Creative Commons license and your intended use is not permitted by statutory regulation or exceeds the permitted use, you will need to obtain permission directly from the copyright holder.

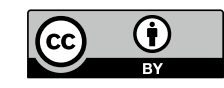

